\title{
Stratum corneum lipids: specificity, role, deficiencies and modulation
}

\author{
Isabelle CASTIEL-HIGOUNENC \\ M. CHOPART \\ C. FERRARIS \\ L'Oréal Recherche, Coordination scientifique, \\ 73, avenue R. Feraud 06200 Nice \\ <icastiel@rd.loreal.com>
}

\begin{abstract}
Lipids are important constituents of the human epidermis. Either free and organized into broad lipid bilayers in the intercorneocytes spaces, or covalently bound to the corneocyte envelope, they play a crucial role in permeability barrier function. This article presents the structures of various human skin ceramides, their role in the maintenance of skin barrier function and homeostasis, and their qualitative and quantitative changes in some cases of dry skin or atopic xerosis. Moreover, we show that reconstructed human skin models could provide valuable tools to assess in vitro the biological interest of active compounds on epidermal lipogenesis. Based on such in vitro studies, we assume that the association of vitamin C and some exogenous sphingolipid could enhance the endogenous ceramide content deficient in some cases of atopic dry skin.
\end{abstract}

Key words: lipids, ceramide, skin barrier function, vitamin C, atopy, dry skin

3-ketosphinganin, sphinganin and ceramides (figure 3).

Using high performance thin layer chromatography (HPTLC), ceramide fraction can be divided into eight distinct subgroups of ceramides based on amide linked fatty acid and number of free hydroxyl groups (figure 4). This high diversity is known to be essential for the cera-

\section{domain: Chemical composition, biosynthesis and organization}

Lipids are important components of human epidermis. During the skin differentiation process, phospholipids, which are major constituents of cell membranes, are progressively transformed into free fatty acids, glycosphingolipids and ceramides (figure 1). Epidermal differentiation is also characterized by the synthesis of specific proteins involved in the formation of the keratinic structure and the corneocyte envelope. These transformations are associated with the formation of an epidermal $\mathrm{pH}$ and calcium gradient in the epidermis.

In the stratum corneum, ceramides are present in an equimolar ratio with free fatty acids and cholesterol. Following exocytose of lamellar bodies in the upper layer of stratum granulosum, ceramides form intercellular membrane bilayers, and, in turn play a crucial role in permeability barrier homeostasis (figure 2). It has been shown that qualitative and/or quantitative changes in stratum corneum lipids may result in defective barrier function, impaired water-retention function, and higher penetration of exogenous compounds through the skin and appearance of dry skin.

Ceramides are sphingolipids that consist of a long-chain of amino alcohol (sphingenin, sphinganin or phytosphingosin bases) to which a hydroxylated or non-hydroxylated long-chain fatty acid is linked via an amide bond. Human skin ceramides are biosynthesized in the epidermis by condensation of serine with palmitoyl-coA to produce successively

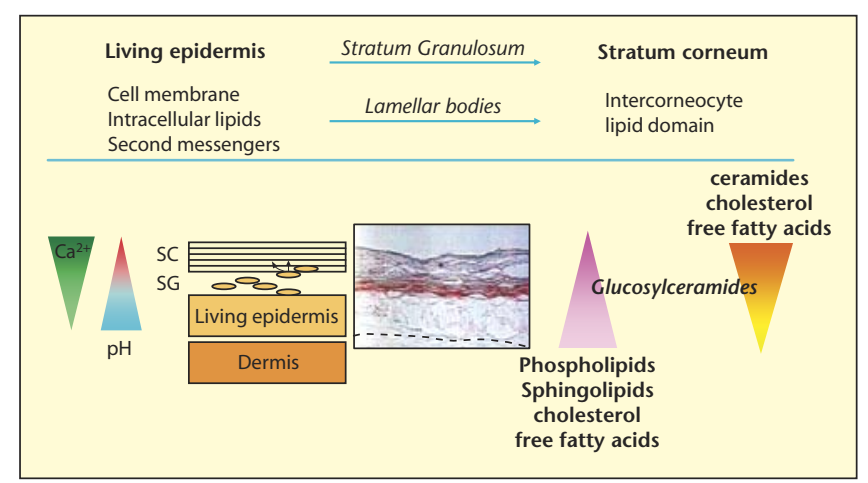

Figure 1. Epidermal differentiation : formation of the intercorneocyte lipid domain.

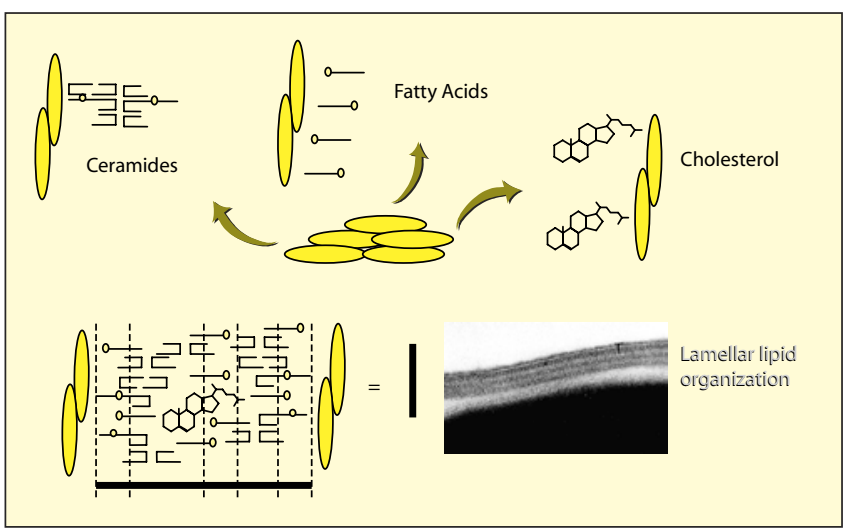

Figure 2. Organization of the intercorneocyte lipid domain. 


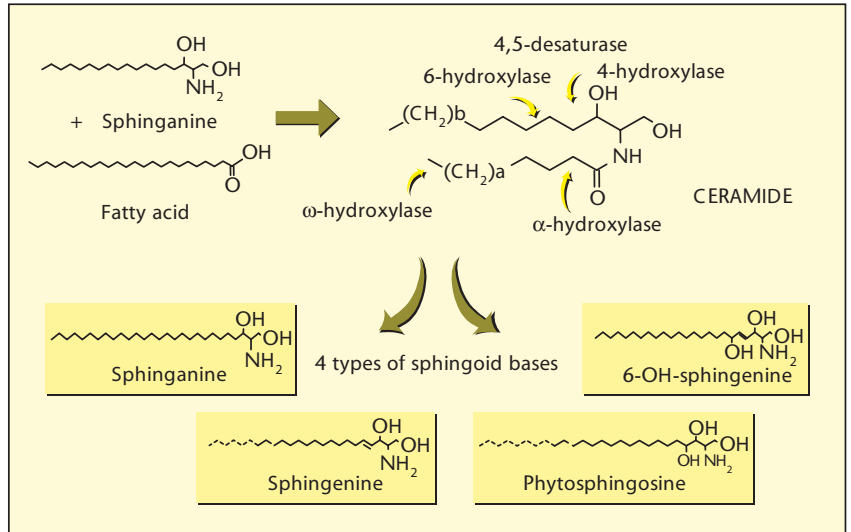

Figure 3. Ceramides biosynthesis.

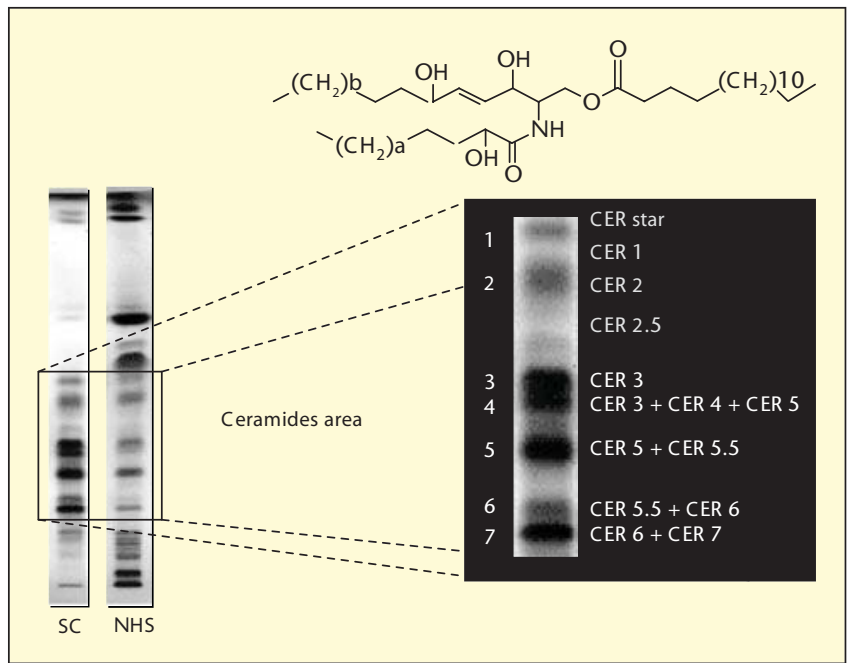

Figure 4. Free ceramides in human skin. M. Chopart, I. Castiel-higounenc, E. Arbey, C. Guey, Q. Gaetani, R. Schmidt, 2001

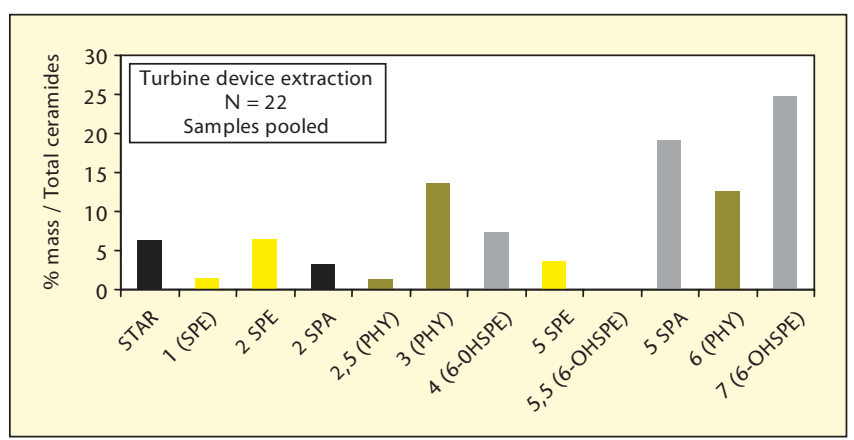

Figure 5. Total sphingoid bases profile of free ceramides. In normal human skin the major sphingoid bases are 6-OH-SPE and PHY. M. Chopart, I. Castiel-higounenc, E. Arbey, C. Guey, Q. Gaetani, R. Schmidt, 2001.

mide multi-lamellae organization and thus for barrier function property of the skin.

Recently, we determined a new profile of human stratum corneum ceramide including two new classes. The first one, counting for about $1.5 \%$ in mass of total ceramides named ceramide 2.5 , consists of a very long chain a-hydroxyacid in amide linkage with a phytosphingosine base with an unsaturated fatty acid (mostly linoleic) on the terminal hydroxyl group. The second one named "star" counting for about $6 \%$, consist of a tri-branch configuration ceramide containing a sphingoid base (mostly 6-OH-4-sphingenine) linked via an amide bond to an a-hydroxylated long chain fatty acid and via an ester bond to a second fatty acid (mostly palmitic) (figure 5) [1].

In the stratum corneum, ceramide can be free or covalently bound to the corneocyte envelope. Stratum corneum free and bound lipids are known to participate to epidermal barrier function: free lipids multi-lamellae are oriented in parallel to the corneocyte surface thanks to bound lipids, together forming the intercellular concrete of the stratum corneum. Omega hydroxyceramides are the predominant covalently bound lipid species of the corneocyte lipid envelope in the epidermis. They are mainly composed of CER A ( $\alpha$-hydroxyacid linked to sphingenine) and CER $B$ ( $\alpha$-hydroxyacid linked to 6-OH-4 sphingenine). We recently identify the presence of two novel types of bound ceramides, one that is composed of a sphinganine base, the second displaying a phytosphingosine (PHY) base and both supposedly linked to an $\alpha$-hydroxyacid [2].

\section{Lipids and barrier function: role and regulation by epidermal homeostasis}

Barrier function depends on several biological processes involved in epidermal homeostasis, such as proliferation, differentiation and lipogenesis. The barrier properties of the skin rely on the importance of the hydrophobic interstitial domain in stratum corneum which is filled with broad bilayers of uninterrupted lipid lamellae. The lamellar structure results from the accumulation and reorganization of lipid-rich organelles, lamellar bodies, which are excreted into intercellular spaces at the interface between stratum corneum and stratum granulosum. This specific lipid organization has been reported to be responsible for the barrier function [3], and the loss of the lamellar structure after solvent extraction or its absence in specific skin disorders, such as free fatty acid deficiency, is associated with breakdown of the barrier properties [4-6].

Cutaneous barrier function is a dynamic system. In one hand, epidermal barrier status regulates exogenous compound penetration through the skin, water flux (TEWL), water tank and thereby enzymatic activities within the stratum corneum. On the other hand TEWL regulates de novo lipid synthesis and epidermal cellular turn over which themselves lead to the repair of the skin barrier.

This has been shown in vitro on reconstructed human epidermis using immunostaining technics to detect Ki67 and MCG (membrane coating granules) on histological sections. Already 5 hours after barrier disruption with a solvent 
we observed an increase of keratinocyte proliferation as shown by Ki67 staining. Release of lipids into intercellular spaces at the interface of stratum granulosum and corneum was shown by MCG staining (figure 6). Using C14 Acetate to follow lipogenesis in vitro, we found that this repair process after barrier disruption was associated with an increased epidermal lipid synthesis (figure 7). Thus, increased water flux following barrier disruption elicits a repair response which induces lipid processing, lipid synthesis and cell proliferation.

\section{Xerosis, atopy and lipids: Epidermal lipid status}

Dry skin is characterized by a deficiency in stratum corneum ceramides, often correlated to an impaired barrier function. Deficiencies are mostly observed in 6-OH-4-sphingenine and Phytosphingosine containing ceramides, which are in majority in human stratum corneum. In Dry skin, the total bound ceramides are deficient of about $30 \%$. All major classes of bound ceramides (CER A; B and phyto) show identical deficiency, and more specifically CER B (REF) [7].

Atopic dermatitis, which is one of the most common forms of chronic eczema, is characterized by dry skin, high frequency of bacterial infection, hyper-responsiveness to environmental stimuli and increased incidence of irritant contact dermatitis. Furthermore, atopic dermatitis has been shown to be accompanied by alterations in the epidermal barrier function including reduced water content and an augmentation in transepidermal water loss (TEWL). Studies have suggested that qualitative and quantitative deficiencies in certain epidermal lipids of intercorneocyte spaces may result in the functional abnormalities observed in atopic patients. Ceramides, which are essential for the structure and the stability of stratum corneum lipid sheets, have been shown to be reduced in atopic dermatitis and ceramide metabolism abnormalities are thought to be etiological factor for dry and barrier disrupted skin.

Keeping this information in mind, many attempts have been made to elucidate the biochemical mechanisms involved in the ceramide deficiency observed in atopic dermatitis. Some studies have reported disturbed lipid processing in the intercellular spaces and abnormal expression of enzymes involved in ceramide synthetic or hydrolytic pathways. Recent studies, however, have challenged these findings indicating that barrier alterations observed in atopic dermatitis are dependent upon the skin zone evaluated as well as the clinical characteristics of subjects being studied.

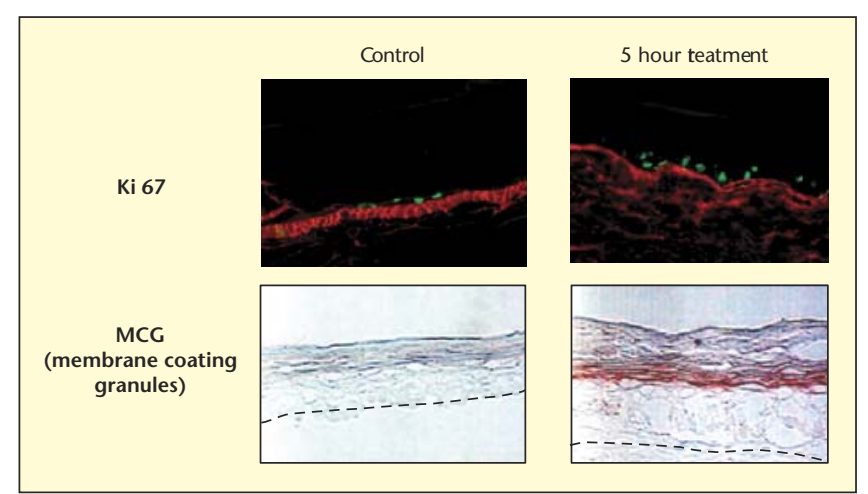

Figure 6. Modification of barrier homeostasis induces proliferation and lipid processing. Study on Reconstructed Human skin - Isabelle Castiel - 1999.

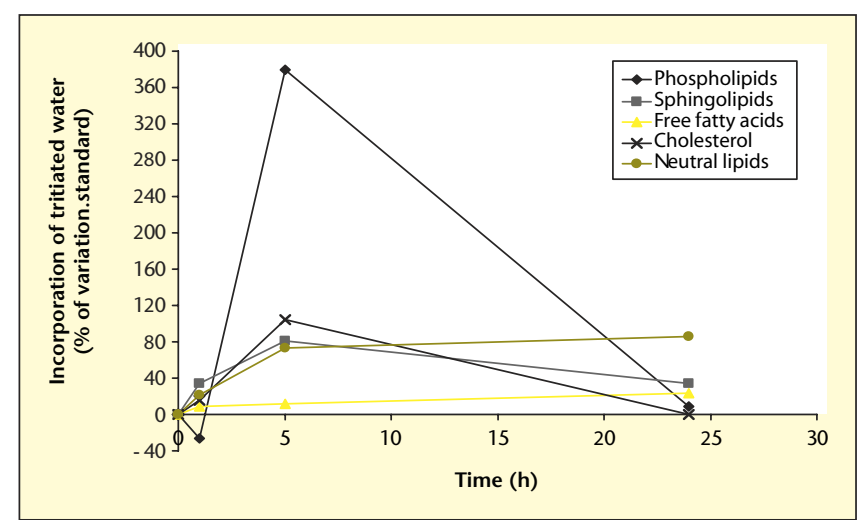

Figure 7. Barrier disruption stimulates lipid de novo synthesis. Study on Reconstructed Human skin - Isabelle Castiel $-1999$.

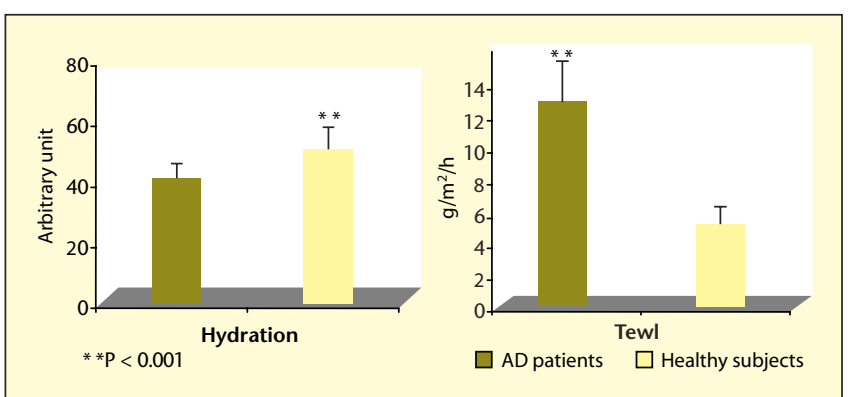

Figure 8. Some atopic xerosis patient $(A D)$ display barrier function disorders.

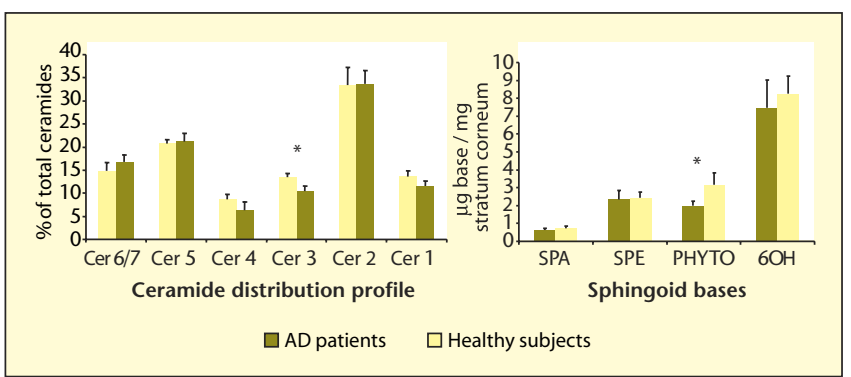

Figure 9. Impaired barrier function in atopic xerosis patients $(A D)$ correlates with abnormal ceramides content. 


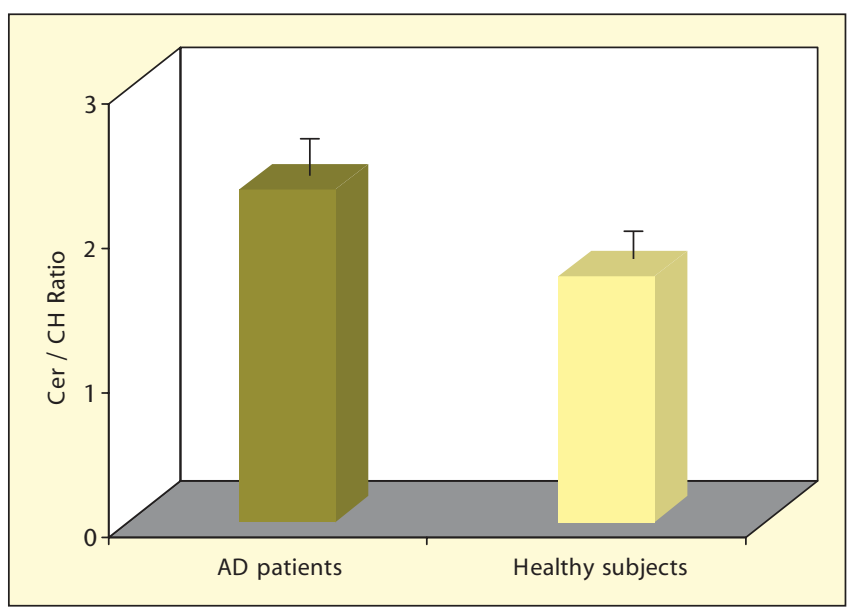

Figure 10. Atopic xerosis patients $(A D)$ with impaired barrier function display a decreased ceramide/cholesterol ratio.

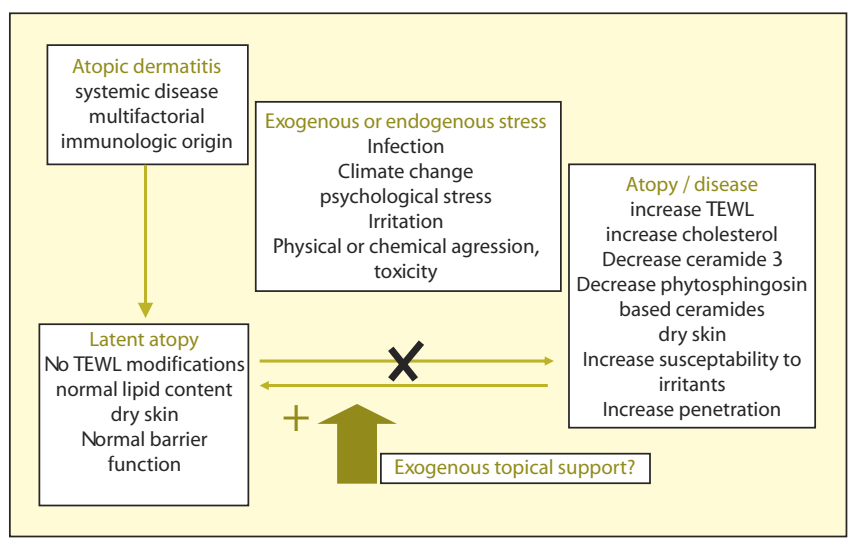

Figure 11. Atopy, lipids and barrier function.

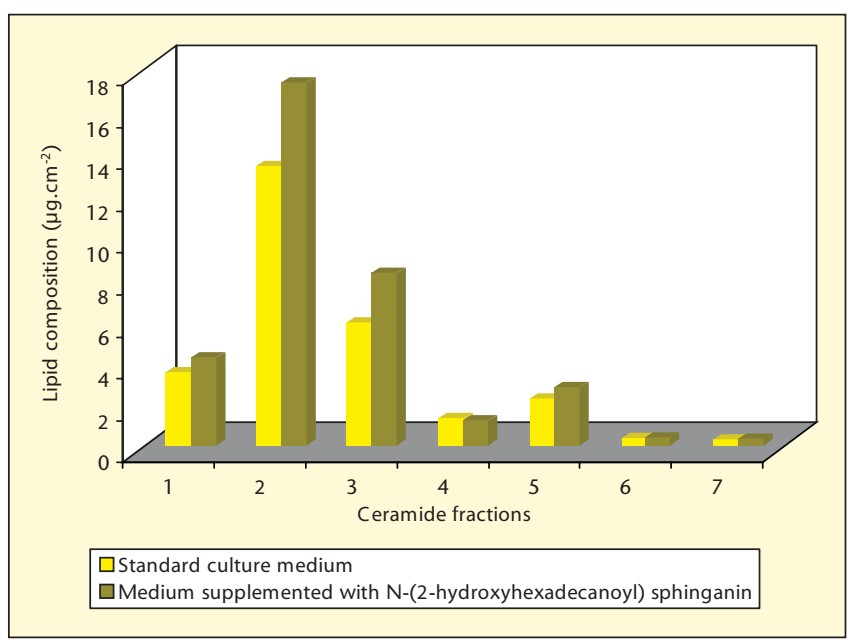

Figure 12. Exogenous sphinganin derived sphingolipid increases ceramide content in human reconstructed skin.

We analyzed epidermal lipid content in a panel of atopic xerosis patients with no active lesions but increase TEWL (figure 8). Both qualitative and quantitative modifications of epidermal lipid content were observed in these subjects.
We found a decrease of phytosphingosine containing ceramides and more specifically of ceramide 3 (figure 9): this suggest that altered barrier function correlates with ceramide deficiencies in stratum corneum. Moreover, atopic xerosis patients with impaired barrier function display a decreased ceramide/cholesterol ratio (figure 10), showing an increased cholesterol synthesis, which has previously been shown to be a normal repair response to barrier disruption. On the other hand, in a second panel of atopic patients displaying no active lesions and no alterations of TEWL, no specific modification of epidermal lipids could be detected [8]. These results indicate that the barrier function of atopic patients lacking lesions may, in fact, be comparable to that of healthy subjects, exhibiting no functional abnormalities.

These findings highlight the complexity of atopic dermatitis etiology. While ceramide deficiency leading to impaired barrier function may account for a major part of the pathology of atopic dermatitis (through the increased penetration of allergens, irritants or infectious agents), abnormalities in ceramide biosynthesis can not be considered as the only etiologic factor for dry skin in atopic patients. Epidermal lipid deficiencies in atopic patients may not be constitutive but secondary to other disorders. Therefore, one could speculate that by adding exogenous lipids to the skin, it would be possible to prevent latent atopy to develop into disease atopic skin (figure 11).

\section{Modulation \\ of epidermal lipogenesis: \\ Evaluation of active compounds on reconstructed human skin models}

The first reconstructed skin models were performed in the early 80 's by lifting at the airliquid interface, normal keratinocytes cultured on appropriate supports. Air exposed, keratinocytes were able to give rise to a differentiated and multilayered reconstructed epidermis, exhibiting similar properties and structure to those of normal human skin.

According to the major role of sphingolipids in barrier function, we assumed that supplementation of dry skin with a ceramide precursor could improve the sphingolipid content of stratum corneum. Therefore, we investigated the ability of human skin to metabolize a sphinganin derived sphingolipid into endogenous human ceramides. Since human reconstructed epidermis has been shown to provide a valuable model for studying skin lipogenesis $[9,10]$, we decided to investigate the effect of medium supplementation with $\mathrm{N}$-(2-hydroxyhexadecanoyl)sphinganin on the epidermal lipid synthesis and composition of an in vitro skin model, EPISKIN ${ }^{\mathrm{TM}}$.

The reconstructed epidermis supplied after 6 days of culture was grown for 7 to 14 additional days at the air-liquid interface in the presence 
of exogenous sphinganin derived sphingolipid added to the culture medium $\left(10^{-7} \mathrm{M}\right.$ and $10^{-8} \mathrm{M}$ ). Lipid composition was assessed after extraction of epidermal sheets using the Bligh and Dyer [11] method. Extracted lipids were separated by one-dimensional High Performance Thin Layer Chromatography (HPTLC), as described by Ponec [12]. For quantification, authentic standards were run in parallel. The quantification was performed after staining and charring, using a photodensitometer. Lipid synthesis was followed using ${ }^{14} \mathrm{C}$-acetate incorporation into epidermal lipids, HPTLC and autoradiography. Reconstructed epidermis was incubated with ${ }^{14} \mathrm{C}$-acetate, prior to $\mathrm{N}$-(2hydroxyhexadecanoyl) sphinganin treatment. We found that culture medium supplementation with $\mathrm{N}$-(2-hydroxyhexadecanoyl) sphinganin increases total ceramide content of human skin in vitro. De novo lipid synthesis study showed that ceramide increase did not result from specific lipogenesis induction as no change could be detected in $\left[{ }^{14} \mathrm{C}\right]$-acetate incorporation into sphingolipids. The increase in ceramide composition could also not be attributed to the incorporation of exogenous sphinganin in stratum corneum bilayers since no specific increase in $\alpha$-hydroxylated sphinganin occurred. However, specific increase of polar ceramides (fractions 1, 2 and 3) containing sphingenin, sphinganin or phytosphingosin bases, show that the exogenous sphingolipid was transformed into endogenous ceramides (figure 12). This result indicated that the reconstructed epidermis had the ability to metabolize a sphinganin derived sphingolipid [13].

Using similar protocols the biological activity of vitamin $\mathrm{C}$ was evaluated on reconstructed human skin. The reconstructed epidermis $\left(\right.$ EPISKIN $^{\mathrm{TM}}$ ) supplied after 6 days of culture was grown for 7 to 14 additional days at the airliquid interface in the presence of vitamin $\mathrm{C}$ added either to the culture medium $\left(10^{-4} \mathrm{M}\right.$ and $10^{-9} \mathrm{M}$ ), or applied topically. Lipid synthesis and composition were assessed as described above. Furthermore, the percutaneous penetration rates of $\left[7-{ }^{14} \mathrm{C}\right]$-benzoic acid and $\left[1,2,6,7-{ }^{3} \mathrm{H}(\mathrm{N})\right]$-hydrocortisone were determined after 13 days of culture in presence of ascorbic acid.

This in vitro study showed that vitamin $C$ was able to increase specifically, the amount of ceramides containing $\alpha$-hydroxylated-fatty acid and/or 6-OH-sphingenin as sphingoid base. Moreover, penetration studies revealed that this observed sphingolipid synthesis, following ascorbic acid supplementation, directly enhanced the barrier properties of reconstructed human skin (figures 13 and 14) [14]. Based on these primary in vitro data we assumed that such a ceramide precursor might

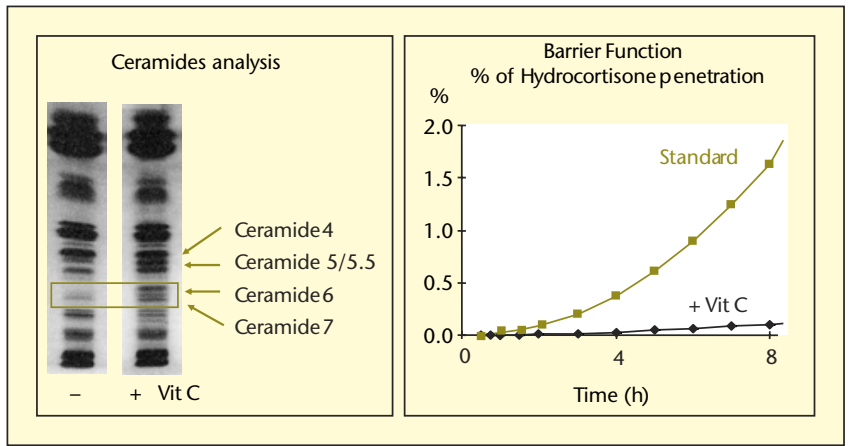

Figure 13. Biological activity of vitamin C on epidermal lipids and skin barrier function in vitro.

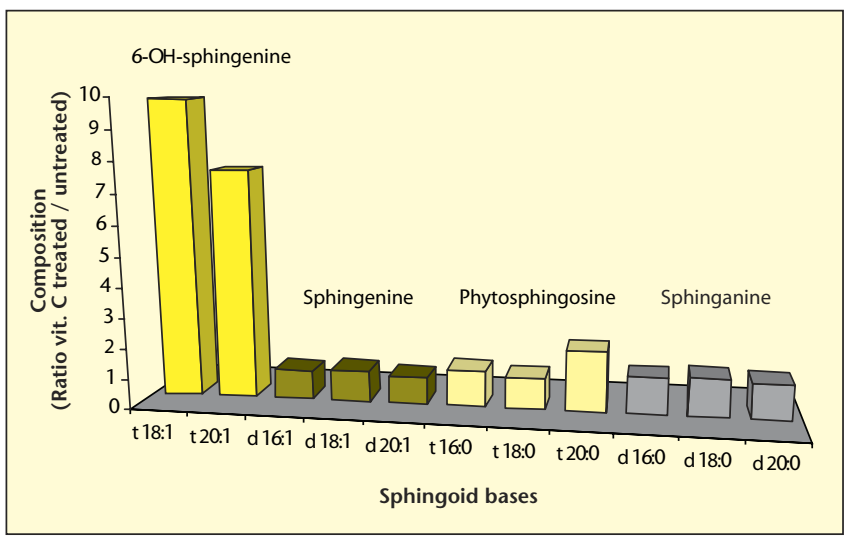

Figure 14. Vitamin C improves 6-hydroxysphingenin epidermal composition in reconstructed skin.

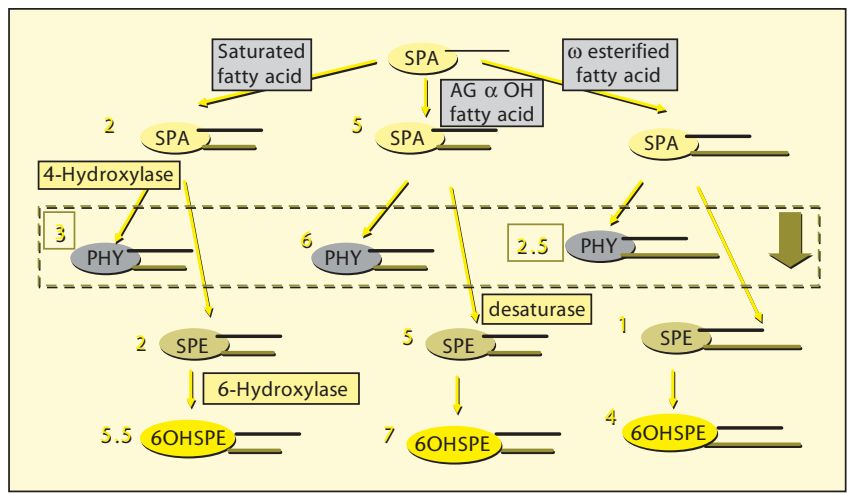

Figure 15. Ceramide synthetic pathway and atopy.

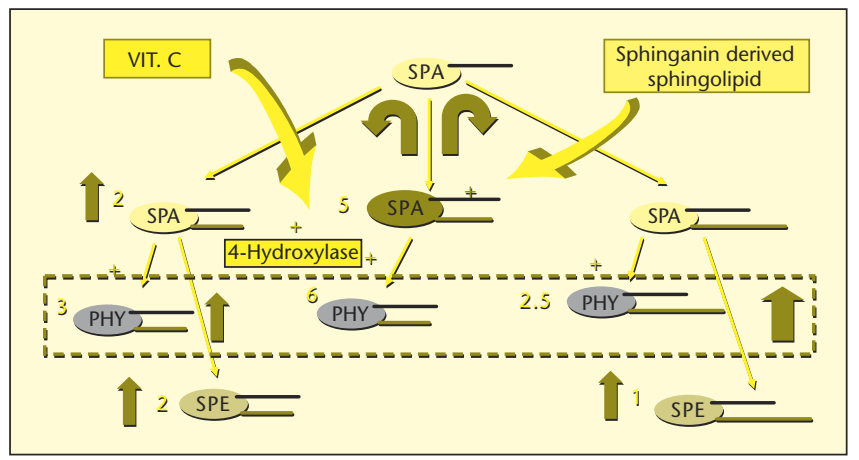

Figure 16. Rationale for exogenous sphingolipid and vitamin C combination in atopy. 
be useful to improve epidermal sphingolipid content in case of stratum corneum lipid deficiencies.

Moreover, we can conclude that reconstructed human skin provides useful tools not only in basic research to better understand the complex mechanisms of keratinocyte differentiation and cell-cell interaction, but also in the safety and efficacy assessment of new dermatocosmetological products. Hence, the biological interest of active compounds for epidermal lipid synthesis can be evaluated in vitro on this model.

\section{Potential contribution of ceramides to atopic skin improvement: association of a sphingoid base and an activator of 4-/6-hydroxylases}

As far as dry atopic skin is concerned we found that disrupted barrier was associated with a decrease of phytosphingosine linked ceramides (figure 15). Since exogenous sphinganin has been shown to improve epidermal ceramide synthesis in vitro and vitamin $\mathrm{C}$ to activate epidermal hydroxylases, we hypothesize that the association of a sphinganine derived sphingolipid and an activator of 4-/6-hydroxylases could provide a useful tool to improve the clinical pattern of atopic patients (figure 16). In conclusion, skin and lipids are closely related to each other. The qualitative and quantitative composition of stratum corneum lipids is shown to be essential for skin integrity and barrier function. Barrier function itself, which is a dynamic system, regulates skin lipogenesis according to the epidermal homeostasis.

In some cases such as xerosis and atopic dermatitis, subjects can display epidermal lipid disorders associated with an impaired barrier function. As shown using in vitro human skin models, it is now possible to evaluate the activity of exogenous compounds such as sphingolipids or vitamins on epidermal lipid synthesis and to hypothesize on the potential contribution of such active molecules on xerosis in vivo.

\section{REFERENCES}

1. CHOPART M, CASTIEL-HIGOUNENC I, ARBEY $\mathrm{E}$, et al. The normal human stratum corneum: $A$ new ceramide profile. (Poster) PPP. Antibes, 2002.

2. CHOPART M, CASTIEL-HIGOUNENCI, SCHMIDT R A new type of covalently bound ceramide in human epithelium. (Poster) Stratum corneum III, Bâle. 2001.

3. ELIAS P, MENON GK. Structural and lipid biochemical correlates of the epidermal permeability barrier. Adv Lipid Res 1991; 24: 1-26.

4. IMOKAWA G, ABE A, IIN K, et al. Decreased level of ceramides in stratum corneum of atopic dermatitis: an etiologic factor in atopic dry skin. J Invest Dermatol 1991; 96: 523-6.

5. WRIGHT $\mathrm{S}$. Essential fatty acids and the skin. $\mathrm{Br}$ I Dermatol 1991; 125: 503-15.

6. HOLLERAN WM, FEINGOLD KR, MAO-QIANG $M$, et al. Regulation of epidermal sphingolipid synthesis by permeability barrier function. / Lipid Res 1991; 32: 1151-8.
7. CHOPART M, CASTIEL-HIGOUNENCI, ARBEY E, et al. Quantitative and qualitative analysis of ceramides in the stratum corneum of normal and dry skin. (Poster) Stratum corneum III. Bâle, 2001.

8. CASTIEL-HIGOUNENCI, JOURDAIN R, FERRARIS $C$, et al. Is barrier function impaired in atopic xerosis? (Poster) EADV, Munich. Octobre 2001.

9. HIGOUNENC I, SPIES F, BODDE H, et al. Lipid composition and barrier function of human skin after grafting onto athymic nude mice. Skin Pharmacol 1994; 7: 167-75.

10. HIGOUNENC I, DEMARCHEZ M, REGNIER M, et al. Improvement of epidermal differentiation and barrier function in reconstructed human skin after grafting onto athymic nude mice. Arch Dermatol Res 1994; 286: 107-14.

11. BLIGH EG, DYER W]. A rapid method of total lipid extraction and purification. Can / Biochem Physiol 1959; 37: 911-7.

12. PONEC M. Lipid metabolism in cultured keratinocytes. Adv Lipid Res 1991; 24: 83-117.

13. CASTIEL-HIGOUNENC I, FERRARIS C, LAVALLE $A$, et al. Exogenous sphinganin derived sphingolipid increases epidermal ceramide content in human reconstructed skin. Nouv Dermatol 1999; 18: 2-11.

14. CASTIEL-HIGOUNENC I, FERRARIS C, GUEY C, et al. Ascorbic acid and derivatives improve 6-hydroxysphingenin synthesis and barrier function of human reconstructed skin via a distinct non-antioxydant related activity. (Poster) ESDR, Montpellier. septembre 1999. 